\title{
Universal acyclic resolutions for arbitrary coefficient groups
}

\author{
by
}

\author{
Michael Levin (Be'er Sheva)
}

\begin{abstract}
We prove that for every compactum $X$ and every integer $n \geq 2$ there are a compactum $Z$ of dimension $\leq n+1$ and a surjective $U V^{n-1}$-map $r: Z \rightarrow X$ such that for every abelian group $G$ and every integer $k \geq 2$ such that $\operatorname{dim}_{G} X \leq k \leq n$ we have $\operatorname{dim}_{G} Z \leq k$ and $r$ is $G$-acyclic.
\end{abstract}

1. Introduction. This paper is devoted to proving the following theorem which was announced in [8].

TheOREM 1.1. Let $X$ be a compactum. Then for every integer $n \geq 2$ there are a compactum $Z$ of dimension $\leq n+1$ and a surjective $U V^{n-1}$ map $r: Z \rightarrow X$ having the property that for every abelian group $G$ and every integer $k \geq 2$ such that $\operatorname{dim}_{G} X \leq k \leq n$ we have $\operatorname{dim}_{G} Z \leq k$ and $r$ is G-acyclic.

The cohomological dimension $\operatorname{dim}_{G} X$ of $X$ with respect to an abelian group $G$ is the least number $n$ such that $\check{H}^{n+1}(X, A ; G)=0$ for every closed subset $A$ of $X$. A space is $G$-acyclic if its reduced Cech cohomology groups modulo $G$ are trivial; a map is $G$-acyclic if every fiber is $G$-acyclic. By the Vietoris-Begle theorem a surjective $G$-acyclic map of compacta cannot raise the cohomological dimension $\operatorname{dim}_{G}$. A compactum $X$ is approximately $n$ connected if any embedding of $X$ into an ANR has the $U V^{n}$-property, i.e. for every neighborhood $U$ of $X$ there is a smaller neighborhood $X \subset V \subset U$ such that the inclusion $V \subset U$ induces the zero homomorphism of the homotopy groups in dimensions $\leq n$. An approximately $n$-connected compactum has trivial reduced Čech cohomology groups in dimensions $\leq n$ with respect to any group $G$. A map is called a $U V^{n}$-map if every fiber is approximately $n$-connected.

Theorem 1.1 generalizes the following results of $[6,7]$.

2000 Mathematics Subject Classification: 55M10, 54F45.

Key words and phrases: cohomological dimension, cell-like and acyclic resolutions. 
THEOREM $1.2([6])$. Let $G$ be an abelian group and let $X$ be a compactum with $\operatorname{dim}_{G} X \leq n, n \geq 2$. Then there are a compactum $Z$ with $\operatorname{dim}_{G} Z \leq n$ and $\operatorname{dim} Z \leq n+1$ and a $G$-acyclic map $r: Z \rightarrow X$ from $Z$ onto $X$.

Theorem $1.3([7])$. Let $X$ be a compactum with $\operatorname{dim}_{\mathbb{Z}} X \leq n \geq 2$. Then there exist a compactum $Z$ with $\operatorname{dim} Z \leq n$ and a cell-like map $r: Z \rightarrow X$ from $Z$ onto $X$ such for every integer $k \geq 2$ and every group $G$ such that $\operatorname{dim}_{G} X \leq k$ we have $\operatorname{dim}_{G} Z \leq k$.

Theorem 1.2 obviously follows from Theorem 1.1. Theorem 1.3 can be derived from Theorem 1.1 as follows. Recall that a compactum is cell-like if any map from the compactum to a CW-complex is null-homotopic. A map is cell-like if its fibers are cell-like. Let $X$ have $\operatorname{dim}_{\mathbb{Z}} X<\infty$ and let $r: Z \rightarrow X$ satisfy the conclusions of Theorem 1.1 for $n=\operatorname{dim}_{\mathbb{Z}} X+1$. Then $\operatorname{dim}_{\mathbb{Z}} Z \leq \operatorname{dim}_{\mathbb{Z}} X \leq n-1$ and because $Z$ is finite-dimensional we have $\operatorname{dim} Z=\operatorname{dim}_{\mathbb{Z}} Z \leq n-1$. Since $r$ is $U V^{n-1}$ and $\operatorname{dim} Z \leq n-1$ we find that $r$ is cell-like. Let $\operatorname{dim}_{G} X \leq k \geq 2$ for a group $G$. If $k \leq n$ then $\operatorname{dim}_{G} Z \leq k$ by Theorem 1.1, and if $k>n$ then $\operatorname{dim}_{G} Z \leq k$ since $\operatorname{dim} Z \leq n-1$. Thus Theorem 1.1 implies Theorem 1.3.

It was observed in [7] that the restriction $k \geq 2$ in Theorem 1.3 cannot be omitted. Therefore Theorem 1.1 does not hold for $k=1$.

Let us discuss possible generalizations of Theorem 1.1. One is tempted to reduce the dimension of $Z$ to $n$. This is partially justified by

TheOREM 1.4 ([8]). Let $X$ be a compactum. Then for every integer $n \geq 2$ there are a compactum $Z$ of dimension $\leq n$ and a surjective $U V^{n-1}-m a p$ $r: Z \rightarrow X$ having the property that for every finitely generated abelian group $G$ and every integer $k \geq 2$ such that $\operatorname{dim}_{G} X \leq k \leq n$ we have $\operatorname{dim}_{G} Z \leq k$ and $r$ is $G$-acyclic.

However, Theorem 1.4 does not hold for arbitrary groups $G$. Indeed, one can show that a $\mathbb{Q}$-acyclic $U V^{1}$-map from a compactum of dimension $\leq 2$ must be $\mathbb{Z}$-acyclic (even cell-like). Thus a compactum $X$ with $\operatorname{dim}_{\mathbb{Z}} X=3$ and $\operatorname{dim}_{\mathbb{Q}} Z=2$ cannot be the image of a compactum of dimension $\leq 2$ under a $\mathbb{Q}$-acyclic $U V^{1}$-map.

The situation becomes more complicated if we drop in Theorem 1.1 the requirement that $r$ is $U V^{n-1}$ and consider

Problem 1.5. Given a compactum $X$, an integer $n \geq 2$ and a collection $\mathcal{G}$ of abelian groups such that $\operatorname{dim}_{G} X \leq n$ for every $G \in \mathcal{G}$, do there exist a compactum $Z$ of dimension $\leq n$ and a $\mathcal{G}$-acyclic surjective map $r: Z \rightarrow X$ such that $\operatorname{dim}_{G} Z \leq \max \left\{\operatorname{dim}_{G} X, 2\right\}$ for every $G \in \mathcal{G}$ ? (The $\mathcal{G}$-acyclicity means the $G$-acyclicity for every $G \in \mathcal{G}$.)

In general the answer to Problem 1.5 is negative [5]. Indeed, let $X$ be a compactum with $\operatorname{dim}_{\mathbb{Z}} X=3, \operatorname{dim}_{\mathbb{Q}} X=2$ and $\operatorname{dim}_{\mathbb{Z}_{p}} X=2$ for every 
prime $p$ and let $G=\mathbb{Q} \oplus(\mathbb{Q} / \mathbb{Z})$. Clearly $\operatorname{dim}_{G} X=2$ and the $G$-acyclicity implies both the $\mathbb{Q}$ - and $(\mathbb{Q} / \mathbb{Z})$-acyclicity. Then it follows from the Bockstein sequence generated by

$$
0 \rightarrow \mathbb{Z} \rightarrow \mathbb{Q} \rightarrow \mathbb{Q} / \mathbb{Z} \rightarrow 0
$$

that the $G$-acyclicity implies the $\mathbb{Z}$-acyclicity and therefore there is no $G$ acyclic resolution for $X$ from a compactum of dimension $\leq 2$.

The situation described in the example can be interpreted in terms of Bockstein theory. Let $\mathcal{G}$ be a collection of abelian groups. Denote by $\sigma(\mathcal{G})$ the union of the Bockstein bases $\sigma(G)$ of all $G \in \mathcal{G}$. Based on the Bockstein inequalities define the closure $\overline{\sigma(\mathcal{G})}$ of $\sigma(\mathcal{G})$ as a collection of abelian groups containing $\sigma(\mathcal{G})$ and possibly some additional groups determined by:

- $\mathbb{Z}_{p} \in \overline{\sigma(\mathcal{G})}$ if $\mathbb{Z}_{p^{\infty}} \in \overline{\sigma(\mathcal{G})}$

- $\mathbb{Z}_{p^{\infty}} \in \overline{\sigma(\mathcal{G})}$ if $\mathbb{Z}_{p} \in \overline{\sigma(\mathcal{G})}$

- $\mathbb{Q} \in \overline{\sigma(\mathcal{G})}$ if $\mathbb{Z}_{(p)} \in \overline{\sigma(\mathcal{G})}$;

- $\mathbb{Z}_{(p)} \in \overline{\sigma(\mathcal{G})}$ if $\mathbb{Q}, \mathbb{Z}_{p^{\infty}} \in \overline{\sigma(\mathcal{G})}$.

One can show that for compact metric spaces the $\mathcal{G}$-acyclicity implies the $\overline{\sigma(\mathcal{G})}$-acyclicity. This motivates the following

Conjecture 1.6. Problem 1.5 can be answered positively if $\operatorname{dim}_{E} X \leq n$ for every $E \in \overline{\sigma(\mathcal{G})}$.

The key open case of this conjecture seems to be constructing a $\mathbb{Q}$-acyclic resolution $r: Z \rightarrow X$ for a compactum $X$ with $\operatorname{dim}_{\mathbb{Q}} X \leq n, n \geq 2$, from a compactum $Z$ of dimension $\leq n$.

2. Preliminaries. All groups are assumed to be abelian, and functions between groups are homomorphisms. $\mathcal{P}$ stands for the set of primes. For a non-empty subset $\mathcal{A}$ of $\mathcal{P}$ let

$$
S(\mathcal{A})=\left\{p_{1}^{n_{1}} p_{2}^{n_{2}} \ldots p_{k}^{n_{k}}: p_{i} \in \mathcal{A}, n_{i} \geq 0\right\}
$$

be the set of positive integers with prime factors from $\mathcal{A}$, and for the empty set define $S(\emptyset)=\{1\}$. Let $G$ be a group and $g \in G$. We say that $g$ is $\mathcal{A}$-torsion if there is $n \in S(\mathcal{A})$ such that $n g=0$, and $g$ is $\mathcal{A}$-divisible if for every $n \in S(\mathcal{A})$ there is $h \in G$ such that $n h=g$. Tor $_{\mathcal{A}} G$ is the subgroup of $\mathcal{A}$-torsion elements of $G$. The group $G$ is $\mathcal{A}$-torsion if $G=\operatorname{Tor}_{\mathcal{A}} G ; G$ is $\mathcal{A}$-torsion free if $\operatorname{Tor}_{\mathcal{A}} G=0$; and $G$ is $\mathcal{A}$-divisible if every element of $G$ is $\mathcal{A}$-divisible.

$G$ is $\mathcal{A}$-local if it is $(\mathcal{P} \backslash \mathcal{A})$-divisible and $(\mathcal{P} \backslash \mathcal{A})$-torsion free. The $\mathcal{A}$ localization of $G$ is the homomorphism $G \rightarrow G \otimes \mathbb{Z}_{(\mathcal{A})}$ defined by $g \mapsto g \otimes 1$, where $\mathbb{Z}_{(\mathcal{A})}=\{n / m: n \in \mathbb{Z}, m \in S(\mathcal{P} \backslash \mathcal{A})\}$. $G$ is $\mathcal{A}$-local if and only if the $\mathcal{A}$-localization of $G$ is an isomorphism. A simply connected $\mathrm{CW}$-complex is 
$\mathcal{A}$-local if its homotopy groups are $\mathcal{A}$-local. A map between two simply connected $\mathrm{CW}$-complexes is an $\mathcal{A}$-localization if the induced homomorphisms of the homotopy and (reduced integral) homology groups are $\mathcal{A}$-localizations.

The extensional dimension of a compactum $X$ is said not to exceed a CW-complex $K$, written e-dim $X \leq K$, if for every closed subset $A$ of $X$ and every map $f: A \rightarrow K$ there is an extension of $f$ over $X$. It is well known that $\operatorname{dim} X \leq n$ is equivalent to e- $\operatorname{dim} X \leq \mathbb{S}^{n}$, and $\operatorname{dim}_{G} X \leq n$ is equivalent to e-dim $X \leq K(G, n)$, where $K(G, n)$ is an Eilenberg-Mac Lane complex of type $(G, n)$.

A map between CW-complexes is said to be combinatorial if the preimage of every subcomplex of the range is a subcomplex of the domain. Let $M$ be a simplicial complex and let $M^{[k]}$ be the $k$-skeleton of $M$ (= the union of all simplexes of $M$ of dimension $\leq k)$. By a resolution of $M$ we mean a $\mathrm{CW}$ complex $E W(M, k)$ and a combinatorial map $\omega: E W(M, k) \rightarrow M$ such that $\omega$ is 1-to-1 over $M^{[k]}$. Let $f: N \rightarrow K$ be a map of a subcomplex $N$ of $M$ into a CW-complex $K$. The resolution is said to be suitable for $f$ if $\left.f \circ \omega\right|_{\omega^{-1}(N)}$ extends to a map $f^{\prime}: E W(M, k) \rightarrow K$. We call $f^{\prime}$ a resolving map for $f$. The resolution is said to be suitable for a compactum $X$ if e-dim $X \leq \omega^{-1}(\Delta)$ for every simplex $\Delta$ of $M$. Note that if $\omega: E W(M, k) \rightarrow M$ is a resolution suitable for $X$ then for every map $\phi: X \rightarrow M$ there is a map $\psi: X \rightarrow E W(M, k)$ such that $(\omega \circ \psi)\left(\phi^{-1}(\Delta)\right) \subset \Delta$ for every simplex $\Delta$ of $M$. We call $\psi$ a combinatorial lifting of $\phi$.

Let $M$ be a finite simplicial complex and let $f: N \rightarrow K$ be a cellular map from a subcomplex $N$ of $M$ to a CW-complex $K$ such that $M^{[k]} \subset N$. A standard way of constructing a resolution suitable for $f$ is described in [7]. Such a resolution $\omega: E W(M, k) \rightarrow M$ is called the standard resolution of $M$ for $f$ and it has the following properties:

- $E W(M, k)$ is $(k-1)$-connected if so are $M$ and $K$;

- $\omega$ is a surjective map and for every simplex $\Delta$ of $M, \omega^{-1}(\Delta)$ is either contractible or homotopy equivalent to $K$;

- for every subcomplex $T$ of $M,\left.\omega\right|_{\omega^{-1}(T)}: E W(T, k)=\omega^{-1}(T) \rightarrow T$ is the standard resolution of $T$ for $\left.f\right|_{N \cap T}: N \cap T \rightarrow K$.

Let $G$ be a group, let $\alpha: L \rightarrow M$ be a surjective combinatorial map of a CW-complex $L$ and a finite simplicial complex $M$, and let $n$ be a positive integer such that $\widetilde{H}_{i}\left(\alpha^{-1}(\Delta) ; G\right)=0$ for every $i<n$ and every simplex $\Delta$ of $M$. One can show by induction on the number of simplexes of $M$ using the Mayer-Vietoris sequence and the Five Lemma that $\alpha_{*}: \widetilde{H}_{i}(L ; G) \rightarrow$ $\widetilde{H}_{i}(M ; G)$ is an isomorphism for $i<n$. We will refer to this fact as the combinatorial Vietoris-Begle theorem.

Proposition 2.1 ([2]). Let $G$ be a group and $p \in \mathcal{P}$. The following conditions are equivalent: 
- $G$ is p-divisible;

- $\operatorname{Ext}\left(\mathbb{Z}_{p} \infty, G\right)$ is p-divisible;

- $\operatorname{Ext}\left(\mathbb{Z}_{p^{\infty}}, G\right)=0$.

Proposition 2.2. Let $G$ be a group, let $2 \leq k \leq n$ be integers and let $\mathcal{F} \subset \mathcal{P}$ and $p \in \mathcal{P} \backslash \mathcal{F}$. Let $M$ be an $(n-1)$-connected finite simplicial complex such that $H_{n}(M)$ is $\mathcal{F}$-torsion, and let $\omega: L=E W(M, k) \rightarrow M$ be the standard resolution of $M$ for a cellular map $f: N \rightarrow K(G, k)$ from a subcomplex $N$ of $M$ containing $M^{[k]}$. Then $L$ is $(k-1)$-connected and for every $1 \leq i \leq n-1$ :

(i) $\pi_{i}(L)$ and $\pi_{n}(L) / \operatorname{Tor}_{\mathcal{F}} \pi_{n}(L)$ are $p$-torsion if $G=\mathbb{Z}_{p}$;

(ii) $\pi_{i}(L)$ and $\pi_{n}(L) / \operatorname{Tor}_{\mathcal{F}} \pi_{n}(L)$ are $p$-torsion and $\pi_{k}(L)$ is p-divisible if $G=\mathbb{Z}_{p} \infty$;

(iii) $\pi_{i}(L)$ and $\pi_{n}(L) / \operatorname{Tor}_{\mathcal{F}} \pi_{n}(L)$ are $q$-divisible and $\pi_{i}(L)$ is $q$-torsion free for every $q \in \mathcal{P}, q \neq p$ if $G=\mathbb{Z}_{(p)}$;

(iv) $\pi_{i}(L)$ and $\pi_{n}(L) / \operatorname{Tor}_{\mathcal{F}} \pi_{n}(L)$ are $q$-divisible and $\pi_{i}(L)$ is $q$-torsion free for every $q \in \mathcal{P}$ if $G=\mathbb{Q}$.

Proof. Recall that $\omega$ is a combinatorial surjective map, $\omega^{-1}(\Delta)$ is either contractible or homotopy equivalent to $K(G, k)$ for every simplex $\Delta$ of $M$, and $L$ is $(k-1)$-connected because so are $M$ and $K(G, k)$. Since $M$ is $(n-1)$-connected and $H_{n}(M)$ is $\mathcal{F}$-torsion we have $H_{n}(M ; \mathbb{Q})=0$ and $H_{n}\left(M ; \mathbb{Z}_{q}\right)=0, H_{n}\left(M ; \mathbb{Z}_{(q)}\right)=0$ for $q \in \mathcal{P} \backslash \mathcal{F}$ and $H_{n}\left(M ; \mathbb{Z}_{q \infty}\right)=0$ for every $q \in \mathcal{P}$.

(i) By the generalized Hurewicz theorem $\widetilde{H}_{*}\left(K\left(\mathbb{Z}_{p}, k\right)\right)$ is $p$-torsion. Then $\widetilde{H}_{*}\left(K\left(\mathbb{Z}_{p}, k\right) ; \mathbb{Q}\right)=0$. Hence by the combinatorial Vietoris-Begle theorem $\widetilde{H}_{i}(L ; \mathbb{Q})=0$ for $i \leq n$ and therefore $\widetilde{H}_{i}(L)$ is torsion for $i \leq n$.

Let $q \in \mathcal{P}$ and $q \neq p$ and $i \leq n-1$. Note that $\widetilde{H}_{*}\left(K\left(\mathbb{Z}_{p}, k\right) ; \mathbb{Z}_{(q)}\right)=0$ and hence by the combinatorial Vietoris-Begle theorem $\widetilde{H}_{i}\left(L ; \mathbb{Z}_{(q)}\right)=0$. Then $\widetilde{H}_{i}(L) \otimes \mathbb{Z}_{(q)}=0$. Thus $\widetilde{H}_{i}(L)$ is torsion and $q$-torsion free and hence $\widetilde{H}_{i}(L)$ is $p$-torsion.

Now let $q \in \mathcal{P} \backslash \mathcal{F}$ and $q \neq p$. Recall $H_{n}\left(M ; \mathbb{Z}_{(q)}\right)=0$. Then using the previous argument we conclude that $H_{n}(L)$ is $q$-torsion free and hence $H_{n}(L)$ is $(\mathcal{F} \cup\{p\})$-torsion.

By the generalized Hurewicz theorem $\pi_{i}(L)$ is $p$-torsion for $i \leq n-1$ and $\pi_{n}(L)$ is $(\mathcal{F} \cup\{p\})$-torsion. Thus $\pi_{n}(L) / \operatorname{Tor}_{\mathcal{F}} \pi_{n}(L)$ is $p$-torsion and (i) follows.

(ii) The argument used in (i) applies to show that $\pi_{i}(L), i \leq n-1$, and $\pi_{n}(L) / \operatorname{Tor}_{\mathcal{F}} \pi_{n}(L)$ are $p$-torsion. Note that $\pi_{k}(L)=H_{k}(L)$. We will show that $H_{k}(L)$ is $p$-divisible and this will imply (ii). Observe that $H_{k}\left(K\left(\mathbb{Z}_{p^{\infty}}, k\right) ; \mathbb{Z}_{p}\right)=\mathbb{Z}_{p^{\infty}} \otimes \mathbb{Z}_{p}=0$. Then since $H_{k}\left(M ; \mathbb{Z}_{p}\right)=0$ the combi- 
natorial Vietoris-Begle theorem implies that $H_{k}\left(L ; \mathbb{Z}_{p}\right)=0$. Thus $H_{k}(L) \otimes$ $\mathbb{Z}_{p}=0$ and therefore $H_{k}(L)$ is $p$-divisible.

(iii) Since $\mathbb{Z}_{(p)}$ is $p$-local we deduce that $\widetilde{H}_{*}\left(K\left(\mathbb{Z}_{(p)}, k\right)\right)$ is $p$-local and therefore $\widetilde{H}_{*}\left(K\left(\mathbb{Z}_{(p)}, k\right) ; \mathbb{Z}_{q}\right)=\widetilde{H}_{*}\left(K\left(\mathbb{Z}_{(p)}, k\right) ; \mathbb{Z}_{q^{\infty}}\right)=0$ for every $q \in \mathcal{P}$, $q \neq p$.

Let $q \in \mathcal{P}, q \neq p$. Recall that $\widetilde{H}_{i}\left(M ; \mathbb{Z}_{q^{\infty}}\right)=0$ for $i \leq n$. Then by the combinatorial Vietoris-Begle theorem $\widetilde{H}_{i}\left(L ; \mathbb{Z}_{q^{\infty}}\right)=0$ for $i \leq n$. Hence by the universal coefficient theorem $\widetilde{H}_{i}(L) * \mathbb{Z}_{q^{\infty}}=0$ and $\widetilde{H}_{i}(L) \otimes \mathbb{Z}_{q^{\infty}}=0$ for $i \leq n-1$ and therefore $\widetilde{H}_{i}(L)$ is $q$-torsion free and $q$-divisible for $i \leq n-1$.

Let $q \in \mathcal{P}, q \neq p$ and $q \notin \mathcal{F}$. Recall that $H_{n}\left(M ; \mathbb{Z}_{q}\right)=0$. By the combinatorial Vietoris-Begle theorem $H_{n}\left(L ; \mathbb{Z}_{q}\right)=0$. Hence $H_{n}(L) \otimes \mathbb{Z}_{q}=0$ and therefore $H_{n}(L)$ is $q$-divisible.

Let $q \in \mathcal{P}, q \neq p$ and $q \in \mathcal{F}$. Then $H_{n}\left(M ; \mathbb{Z}_{q \infty}\right)=0$. By the combinatorial Vietoris-Begle theorem $H_{n}\left(L ; \mathbb{Z}_{q^{\infty}}\right)=0$. Hence $H_{n}(L) \otimes \mathbb{Z}_{q^{\infty}}=0$ and therefore $H_{n}(L) / \operatorname{Tor}_{q} H_{n}(L)$ is $q$-divisible.

Now using completion and localization theories [1] we will pass to the homotopy groups of $L$.

Let $q \in \mathcal{P}$. Define $\mathcal{A}=\mathcal{P} \backslash\{q\}$. Let $\alpha: L \rightarrow L_{\alpha}$ be an $\mathcal{A}$-localization of $L$. Recall that $\widetilde{H}_{i}(L)$ is $q$-torsion free and $q$-divisible for $i \leq n-1$. Then $\alpha$ induces an isomorphism of the groups $\widetilde{H}_{*}(L)$ and $\widetilde{H}_{*}\left(L_{\alpha}\right)$ in dimensions $\leq n-1$. Hence by the Whitehead theorem, $\alpha$ induces an isomorphism of the homotopy groups in dimensions $\leq n-1$ and therefore $\pi_{i}(L)$ is $\mathcal{A}$-local (that is, $q$-divisible and $q$-torsion free) for $i \leq n-1$.

Let $q \in \mathcal{P}, q \neq p$ and $q \notin \mathcal{F}$. Let $\beta: L \rightarrow L_{\beta}$ be a $q$-completion of $L$. Then $\beta$ induces an isomorphism of $\widetilde{H}_{*}\left(L ; \mathbb{Z}_{q}\right)$ and $\widetilde{H}_{*}\left(L_{\beta} ; \mathbb{Z}_{q}\right) ;$ since $H_{n}\left(L ; \mathbb{Z}_{q}\right)=0$ we get $H_{n}\left(L_{\beta} ; \mathbb{Z}_{q}\right)=0$ and therefore $H_{n}\left(L_{\beta}\right)$ is $q$-divisible. Now since $\pi_{i}(L)$ is $q$-divisible and $q$-torsion free we have $\operatorname{Hom}\left(\mathbb{Z}_{q^{\infty}}, \pi_{i}(L)\right)=0, i \leq n-1$, and by Proposition $2.1, \operatorname{Ext}\left(\mathbb{Z}_{q^{\infty}}, \pi_{i}(L)\right)=0, i \leq n-1$. Then the exact sequence

$$
0 \rightarrow \operatorname{Ext}\left(\mathbb{Z}_{q^{\infty}}, \pi_{i}(L)\right) \rightarrow \pi_{i}\left(L_{\beta}\right) \rightarrow \operatorname{Hom}\left(\mathbb{Z}_{q^{\infty}}, \pi_{i-1}(L)\right) \rightarrow 0
$$

implies that $L_{\beta}$ is $(n-1)$-connected and $\operatorname{Ext}\left(\mathbb{Z}_{q^{\infty}}, \pi_{n}(L)\right)=\pi_{n}\left(L_{\beta}\right)$. Thus $\pi_{n}\left(L_{\beta}\right)=H_{n}\left(L_{\beta}\right)$ and hence $\operatorname{Ext}\left(\mathbb{Z}_{q^{\infty}}, \pi_{n}(L)\right)$ is $q$-divisible. Then by Proposition 2.1, $\pi_{n}(L)$ is $q$-divisible and therefore $\pi_{n}(L) / \operatorname{Tor}_{q} \pi_{n}(L)$ is $q$-divisible.

Now assume that $q \in \mathcal{F}$. Once again let $\mathcal{A}=\mathcal{P} \backslash\{q\}$ and let $\alpha: L \rightarrow L_{\alpha}$ be an $\mathcal{A}$-localization of $L$. Recall that $\widetilde{H}_{i}(L)$ is $q$-torsion free and $q$-divisible for $i \leqq n-1$ and therefore $\alpha$ induces an isomorphism of the groups $\widetilde{H}_{*}(L)$ and $\widetilde{\widetilde{H}}_{*}\left(L_{\alpha}\right)$ in dimensions $\leq n-1$. Note that $H_{n}(L) \otimes \mathbb{Z}_{(\mathcal{A})}=H_{n}(L) \otimes$ $\mathbb{Z}[1 / q]=\left(H_{n}(L) / \operatorname{Tor}_{q} H_{n}(L)\right) \otimes \mathbb{Z}[1 / q]$ and hence since $H_{n}(L) / \operatorname{Tor}_{q} H_{n}(L)$ is $q$-divisible we infer that the $\mathcal{A}$-localization of $H_{n}(L)$ is an epimorphism. Then by the Whitehead theorem $\alpha$ induces an epimorphism of the $n$th ho- 
motopy groups of $L$ and $L_{\alpha}$ and therefore the $\mathcal{A}$-localization of $\pi_{n}(L)$ is an epimorphism. This happens only if $\pi_{n}(L) / \operatorname{Tor}_{q} \pi_{n}(L)$ is $q$-divisible, and (iii) is proved.

(iv) The proof is similar to the proof of (iii).

Let $X$ be a compactum and let $n$ be a positive integer. The Bockstein basis of abelian groups is the collection $\sigma=\left\{\mathbb{Q}, \mathbb{Z}_{p}, \mathbb{Z}_{p^{\infty}}, \mathbb{Z}_{(p)}: p \in \mathcal{P}\right\}$ of groups. Define the Bockstein basis of $X$ in dimensions $\leq n$ as $\sigma(X, n)=$ $\left\{E \in \sigma: \operatorname{dim}_{E} X \leq n\right\}$. Following [6] define:

$$
\begin{aligned}
& \mathcal{T}(X, n)=\left\{p \in \mathcal{P}: \mathbb{Z}_{p} \text { or } \mathbb{Z}_{p^{\infty}} \in \sigma(X, n)\right\} \\
& \mathcal{D}(X, n)= \begin{cases}\emptyset & \text { if } \sigma(X, n) \text { contains only torsion groups, } \\
\mathcal{P} & \text { if } \mathbb{Q} \in \sigma(X, n) \text { but } \mathbb{Z}_{(p)} \in \sigma(X, n) \text { for no } p \in \mathcal{P}, \\
\mathcal{P} \backslash\left\{p \in \mathcal{P}: \mathbb{Z}_{(p)} \in \sigma(X, n)\right\} \text { otherwise; }\end{cases} \\
& \mathcal{F}(X, n)=\mathcal{D}(X, n) \backslash \mathcal{T}(X, n) .
\end{aligned}
$$

Note that for every group $G$ such that $\operatorname{dim}_{G} X \leq n, G$ is $\mathcal{F}(X, n)$-torsion free.

Proposition 2.3. Let $X$ be a compactum such that $\mathcal{D}(X, n) \neq \emptyset$. Then $\operatorname{dim}_{H} X \leq n$ for every group $H$ such that $H$ is $\mathcal{D}(X, n)$-divisible and $\mathcal{F}(X, n)$ torsion free.

Proof. Let $G=\bigoplus\{E: E \in \sigma(X, n)\}$. Then $\operatorname{dim}_{G} X \leq n$. One can easily verify that in the notations of Proposition 2.4 of $[6], \mathcal{D}(G)=\mathcal{D}(X, n)$ and $\mathcal{F}(G)=\mathcal{F}(X, n)$. Then the result follows from Proposition 2.4 of $[6]$.

In the proof of Theorem 1.1 we will also use the following facts.

Proposition 2.4 ([7]). Let $K$ be a simply connected $C W$-complex such that $K$ has only finitely many non-trivial homotopy groups. Let $X$ be a compactum such that $\operatorname{dim}_{\pi_{i}(K)} X \leq i$ for $i>1$. Then e-dim $X \leq K$.

Let $K^{\prime}$ be a simplicial complex. We say that maps $h: K \rightarrow K^{\prime}, g: L \rightarrow L^{\prime}$, $\alpha: L \rightarrow K$ and $\alpha^{\prime}: L^{\prime} \rightarrow K^{\prime}$ combinatorially commute if $\left(\alpha^{\prime} \circ g\right)\left((h \circ \alpha)^{-1}(\Delta)\right)$ $\subset \Delta$ for every simplex $\Delta$ of $K^{\prime}$. Recall that a map $h^{\prime}: K \rightarrow L^{\prime}$ is a combinatorial lifting of $h$ to $L^{\prime}$ if $\left(\alpha^{\prime} \circ h^{\prime}\right)\left(h^{-1}(\Delta)\right) \subset \Delta$ for every simplex $\Delta$ of $K^{\prime}$.

For a simplicial complex $K$ and $a \in K$, st $(a)$ denotes the union of all the simplexes of $K$ containing $a$.

Proposition 2.5 ([7]). (i) Let a compactum $X$ be represented as the inverse limit $X=\lim _{K_{i}}$ of finite simplicial complexes $K_{i}$ with bonding maps $h_{j}^{i}: K_{j} \rightarrow K_{i}$. Fix $i$ and let $\omega: E W\left(K_{i}, k\right) \rightarrow K_{i}$ be a resolution of $K_{i}$ which is suitable for $X$. Then there is a sufficiently large $j$ such that $h_{j}^{i}$ admits a combinatorial lifting to $E W\left(K_{i}, k\right)$. 
(ii) Let $h: K \rightarrow K^{\prime}, h^{\prime}: K \rightarrow L^{\prime}$ and $\alpha^{\prime}: L^{\prime} \rightarrow K^{\prime}$ be maps of a simplicial complex $K^{\prime}$ and $C W$-complexes $K$ and $L^{\prime}$ such that $h$ and $\alpha^{\prime}$ are combinatorial and $h^{\prime}$ is a combinatorial lifting of $h$. Then there is a cellular approximation of $h^{\prime}$ which is also a combinatorial lifting of $h$.

(iii) Let $K$ and $K^{\prime}$ be simplicial complexes, let maps $h: K \rightarrow K^{\prime}, g$ : $L \rightarrow L^{\prime}, \alpha: L \rightarrow K$ and $\alpha^{\prime}: L^{\prime} \rightarrow K^{\prime}$ combinatorially commute and let $h$ be combinatorial. Then

$$
g\left(\alpha^{-1}(\operatorname{st}(x))\right) \subset \alpha^{\prime-1}(\operatorname{st}(h(x))) \quad \text { and } \quad h\left(\operatorname{st}((\alpha(z))) \subset \operatorname{st}\left(\left(\alpha^{\prime} \circ g\right)(z)\right)\right.
$$

for every $x \in K$ and $z \in L$.

3. Proof of Theorem 1.1. Write $\mathcal{D}=\mathcal{D}(X, n)$ and $\mathcal{F}=\mathcal{F}(X, n)$. Represent $X$ as the inverse limit $X=\lim _{\longleftarrow}\left(K_{i}, h_{i}\right)$ of finite simplicial complexes $K_{i}$ with combinatorial bonding maps $h_{i+1}: K_{i+1} \rightarrow K_{i}$ and the projections $p_{i}: X \rightarrow K_{i}$ such that $\operatorname{diam}\left(p_{i}^{-1}(\Delta)\right) \leq 1 / i$ for every simplex $\Delta$ of $K_{i}$. Following A. Dranishnikov $[3,4]$ we construct by finite induction CW-complexes $L_{i}$ and maps $g_{i+1}: L_{i+1} \rightarrow L_{i}, \alpha_{i}: L_{i} \rightarrow K_{i}$ such that:

(a) $L_{i}$ is $(n+1)$-dimensional and obtained from $K_{i}^{[n+1]}$ by replacing some $(n+1)$-simplexes by $(n+1)$-cells attached to the boundary of the replaced simplexes by a map of degree $\in S(\mathcal{F})$. Then $\alpha_{i}$ is a projection of $L_{i}$ taking the new cells to the original ones such that $\alpha_{i}$ is 1 -to- 1 over $K_{i}^{[n]}$. We define a simplicial structure on $L_{i}$ for which $\alpha_{i}$ is a combinatorial map and refer to this simplicial structure while constructing resolutions of $L_{i}$. Note that for $\mathcal{F}=\emptyset$ we do not replace simplexes of $K_{i}^{[n+1]}$ at all.

(b) The maps $h_{i+1}, g_{i+1}, \alpha_{i+1}$ and $\alpha_{i}$ combinatorially commute. Recall that this means that $\left(\alpha_{i} \circ g_{i+1}\right)\left(\left(h_{i+1} \circ \alpha_{i+1}\right)^{-1}(\Delta)\right) \subset \Delta$ for every simplex $\Delta$ of $K_{i}$.

We will construct $L_{i}$ in such a way that $Z=\lim _{(}\left(L_{i}, g_{i}\right)$ will admit a map $r: Z \rightarrow X$ such that $Z$ and $r$ satisfy the conclusions of the theorem.

Let $E \in \sigma$ be such that $\operatorname{dim}_{E} X \leq k, 2 \leq k \leq n$, and let $f: N \rightarrow$ $K(E, k)$ be a cellular map from a subcomplex $N$ of $L_{i}$ with $L_{i}^{[k]} \subset N$. Let $\omega_{L}: E W\left(L_{i}, k\right) \rightarrow L_{i}$ be the standard resolution of $L_{i}$ for $f$. We are going to construct from $\omega_{L}$ a resolution $\omega: E W\left(K_{i}, k\right) \rightarrow K_{i}$ of $K_{i}$ suitable for $X$. If $\operatorname{dim} K_{i} \leq k$ set $\omega=\alpha_{i} \circ \omega_{L}: E W\left(K_{i}, k\right)=E W\left(L_{i}, k\right) \rightarrow K_{i}$.

If $\operatorname{dim} K_{i}>k$ set $\omega_{k}=\alpha_{i} \circ \omega_{L}: E W_{k}\left(K_{i}, k\right)=E W\left(L_{i}, k\right) \rightarrow K_{i}$. We will construct by induction resolutions $\omega_{j}: E W_{j}\left(K_{i}, k\right) \rightarrow K_{i}, k+1 \leq j \leq$ $\operatorname{dim} K_{i}$, such that $E W_{j}\left(K_{i}, k\right)$ is a subcomplex of $E W_{j+1}\left(K_{i}, k\right)$ and $\omega_{j+1}$ extends $\omega_{j}$ for every $k \leq j<\operatorname{dim} K_{i}$.

Assume that $\omega_{j}: E W_{j}\left(K_{i}, k\right) \rightarrow K_{i}, k \leq j<\operatorname{dim} K_{i}$, is constructed. For every $(j+1)$-simplex $\Delta$ of $K_{i}$ consider the subcomplex $\omega_{j}^{-1}(\Delta)$ of $E W_{j}\left(K_{i}, k\right)$. Enlarge $\omega_{j}^{-1}(\Delta)$ by attaching $(n+1)$-cells in order to kill the 
elements of $\operatorname{Tor}_{\mathcal{F}} \pi_{n}\left(\omega_{j}{ }^{-1}(\Delta)\right)$, and attaching cells of dimension $>n+1$ in order to get a subcomplex with trivial homotopy groups in dimensions $>n$. Let $E W_{j+1}\left(K_{i}, k\right)$ be $E W_{j}\left(K_{i}, k\right)$ with all the cells attached for all $(j+1)$ simplexes $\Delta$ of $K_{i}$ and let $\omega_{j+1}: E W_{j+1}\left(K_{i}, k\right) \rightarrow K_{i}$ be an extension of $\omega_{j}$ sending the interior points of the attached cells to the interior of the corresponding $\Delta$.

Finally, define $E W\left(K_{i}, k\right)=E W_{j}\left(K_{i}, k\right)$ and $\omega=\omega_{j}: E W_{j}\left(K_{i}, k\right) \rightarrow K_{i}$ for $j=\operatorname{dim} K_{i}$. Note that since we attach cells only of dimension $>n$, the $n$-skeleton of $E W\left(K_{i}, k\right)$ coincides with the $n$-skeleton of $E W\left(L_{i}, k\right)$.

Let us show that $E W\left(K_{i}, k\right)$ is suitable for $X$. Fix a simplex $\Delta$ of $K_{i}$. Since $\omega^{-1}(\Delta)$ is contractible if $\operatorname{dim} \Delta \leq k$, assume that $\operatorname{dim} \Delta>k$. Set $T=\alpha_{i}^{-1}(\Delta)$. Note that it follows from the construction that $T$ is $(n-1)$ connected, $H_{n}(T)$ is $\mathcal{F}$-torsion, $\omega^{-1}(\Delta)$ is $(k-1)$-connected, $\pi_{n}\left(\omega^{-1}(\Delta)\right)=$ $\pi_{n}\left(\omega_{L}^{-1}(T)\right) / \operatorname{Tor}_{\mathcal{F}} \pi_{n}\left(\omega_{L}^{-1}(T)\right), \pi_{j}\left(\omega^{-1}(\Delta)\right)=0$ for $j \geq n+1$ and $\pi_{j}\left(\omega^{-1}(\Delta)\right)$ $=\pi_{j}\left(\omega_{L}^{-1}(T)\right)$ for $j \leq n-1$.

Consider the following cases.

CASE 1: $E=\mathbb{Z}_{p}$. By (i) of Proposition 2.2, $\pi_{j}\left(\omega_{L}^{-1}(T)\right), j \leq n-1$, and $\pi_{n}\left(\omega_{L}^{-1}(T)\right) / \operatorname{Tor}_{\mathcal{F}} \pi_{n}\left(\omega_{L}^{-1}(T)\right)$ are $p$-torsion. Then $\pi_{j}\left(\omega^{-1}(\Delta)\right)$ is $p$-torsion for $j \leq n$. Therefore $\operatorname{dim}_{\pi_{j}\left(\omega^{-1}(\Delta)\right)} X \leq \operatorname{dim}_{\mathbb{Z}_{p}} X \leq k$ for $j \geq k$ and hence by Proposition 2.4, e- $\operatorname{dim} X \leq \omega^{-1}(\Delta)$.

CASE 2: $E=\mathbb{Z}_{p^{\infty}}$. Then by (ii) of Proposition $2.2, \pi_{j}\left(\omega_{L}^{-1}(T)\right), j \leq$ $n-1$, and $\pi_{n}\left(\omega_{L}^{-1}(T)\right) / \operatorname{Tor}_{\mathcal{F}} \pi_{n}\left(\omega_{L}^{-1}(T)\right)$ are $p$-torsion and $\pi_{k}\left(\omega_{L}^{-1}(T)\right)$ is $p$-divisible. Then $\pi_{j}\left(\omega^{-1}(\Delta)\right)$ is $p$-torsion for $j \leq n$ and $\pi_{k}\left(\omega^{-1}(\Delta)\right)$ is $p$-divisible. Therefore by the Bockstein theorem we have the inequalities $\operatorname{dim}_{\pi_{k}\left(\omega^{-1}(\Delta)\right)} X \leq \operatorname{dim}_{\mathbb{Z}_{p} \infty} X \leq k$ and $\operatorname{dim}_{\pi_{j}\left(\omega^{-1}(\Delta)\right)} X \leq \operatorname{dim}_{\mathbb{Z}_{p} \infty} X+1 \leq$ $k+1$ for $j \geq k+1$. Hence e-dim $X \leq \omega^{-1}(\Delta)$ by Proposition 2.4.

CASE 3: $E=\mathbb{Z}_{(p)}$. Then by (iii) of Proposition $2.2, \pi_{j}\left(\omega_{L}^{-1}(T)\right), j \leq$ $n-1$, is $p$-local and $\pi_{n}\left(\omega_{L}^{-1}(T)\right) / \operatorname{Tor}_{\mathcal{F}} \pi_{n}\left(\omega_{L}^{-1}(T)\right)$ is $q$-divisible for every $q \in \mathcal{P}, q \neq p$. Then $\pi_{j}\left(\omega^{-1}(\Delta)\right), j \leq n-1$, is $p$-local and $\pi_{n}\left(\omega^{-1}(\Delta)\right)$ is $\mathcal{D}$-divisible and $\mathcal{F}$-torsion free. Therefore $\operatorname{dim}_{\pi_{j}\left(\omega^{-1}(\Delta)\right)} X \leq k$ for $j \leq n-1$ and by Proposition 2.3, $\operatorname{dim}_{\pi_{n}\left(\omega^{-1}(\Delta)\right)} X \leq n$. Hence e-dim $X \leq \omega^{-1}(\Delta)$ by Proposition 2.4.

Case 4: $E=\mathbb{Q}$. This case is similar to the previous one.

Thus we have shown that $E W\left(K_{i}, k\right)$ is suitable for $X$. Now replacing $K_{i+1}$ by $K_{j}$ with a sufficiently large $j$ we may assume by Proposition 2.5(i) that there is a combinatorial lifting of $h_{i+1}$ to $h_{i+1}^{\prime}: K_{i+1} \rightarrow E W\left(K_{i}, k\right)$. By Proposition 2.5(ii) we replace $h_{i+1}^{\prime}$ by its cellular approximation preserving the property of $h_{i+1}^{\prime}$ of being a combinatorial lifting of $h_{i+1}$. 
Let $\Delta$ be a simplex of $K_{i}$ and let $\tau:\left(\alpha_{i} \circ \omega_{L}\right)^{-1}(\Delta) \rightarrow \omega^{-1}(\Delta)$ be the inclusion. Note that from the construction it follows that the kernel of the induced homomorphism $\tau_{*}: \pi_{n}\left(\left(\alpha_{i} \circ \omega_{L}\right)^{-1}(\Delta)\right) \rightarrow \pi_{n}\left(\omega^{-1}(\Delta)\right)$ is $\mathcal{F}$-torsion. Using this fact and the reasoning in the proof of Theorem 1.2 of [6] one can construct from $K_{i+1}^{[n+1]}$ a CW-complex $L_{i+1}$ by replacing some $(n+1)$-simplexes of $K_{i+1}^{[n+1]}$ by $(n+1)$-cells attached to the boundary of the replaced simplexes by a map of degree $\in S(\mathcal{F})$ such that $h_{i+1}^{\prime}$ restricted to $K_{i+1}^{[n]}$ extends to a map $g_{i+1}^{\prime}: L_{i+1} \rightarrow E W\left(L_{i}, n\right)$ such that $g_{i+1}^{\prime}, \alpha_{i+1}, h_{i+1}$ and $\alpha_{i} \circ \omega_{L}$ combinatorially commute, where $\alpha_{i+1}$ is a projection of $L_{i+1}$ into $K_{i+1}$ taking the new cells to the original ones in such a way that $\alpha_{i+1}$ is 1 -to- 1 over $K_{i+1}^{[n]}$.

Now define $g_{i+1}=\omega_{L} \circ g_{i+1}^{\prime}: L_{i+1} \rightarrow L_{i}$ and finally define a simplicial structure on $L_{i+1}$ for which $\alpha_{i+1}$ is a combinatorial map. It is easy to check that the properties (a) and (b) are satisfied. Since the triangulation of $L_{i+1}$ can be replaced by any of its barycentric subdivisions we may also assume that

(c) $\operatorname{diam} g_{i+1}^{j}(\Delta) \leq 1 / i$ for every simplex $\Delta$ in $L_{i+1}$ and $j \leq i$, where $g_{i}^{j}=g_{j+1} \circ g_{j+2} \circ \ldots \circ g_{i}: L_{i} \rightarrow L_{j}$.

Define $Z=\lim _{(}\left(L_{i}, g_{i}\right)$ and let $r_{i}: Z \rightarrow L_{i}$ be the projections. Clearly $\operatorname{dim} Z \leq n+1$. To construct $L_{i+1}$ we used an arbitrary map $f: N \rightarrow K(E, k)$ such that $E \in \sigma, \operatorname{dim}_{E} X \leq k, 2 \leq k \leq n$ and $N$ is a subcomplex of $L_{i}$ containing $L_{i}^{[k]}$. By a standard reasoning described in detail in the proof of Theorem 1.6 of [7] one can show that choosing $E$ and $f$ in an appropriate way for each $i$ we can achieve that $\operatorname{dim}_{E} Z \leq k$ for every integer $2 \leq k \leq n$ and every $E \in \sigma$ such that $\operatorname{dim}_{\mathbb{Z}_{p}} X \leq k$. Then by the Bockstein theorem $\operatorname{dim}_{G} Z \leq k$ for every group $G$ such that $\operatorname{dim}_{G} X \leq k, 2 \leq k \leq n$.

By Proposition 2.5(iii), properties (a) and (b) imply that for every $x \in X$ and $z \in Z$ the following holds:

(d1) $g_{i+1}\left(\alpha_{i+1}^{-1}\left(\operatorname{st}\left(p_{i+1}(x)\right)\right)\right) \subset \alpha_{i}^{-1}\left(\operatorname{st}\left(p_{i}(x)\right)\right)$,

(d2) $h_{i+1}\left(\operatorname{st}\left(\left(\alpha_{i+1} \circ r_{i+1}\right)(z)\right)\right) \subset \operatorname{st}\left(\left(\alpha_{i} \circ r_{i}\right)(z)\right)$.

Define a map $r: Z \rightarrow X$ by $r(z)=\bigcap\left\{p_{i}^{-1}\left(\operatorname{st}\left(\left(\alpha_{i} \circ r_{i}\right)(z)\right)\right): i=1,2, \ldots\right\}$. Then (d2) implies that $r$ is indeed well defined and continuous.

Properties (d1) and (d2) also imply that for every $x \in X$,

$$
r^{-1}(x)=\lim _{\longleftarrow}\left(\alpha_{i}^{-1}\left(\operatorname{st}\left(p_{i}(x)\right)\right),\left.g_{i}\right|_{\alpha_{i}^{-1}\left(\operatorname{st}\left(p_{i}(x)\right)\right)}\right)
$$

where the map $\left.g_{i}\right|_{\ldots}$ is considered as a map to $\alpha_{i-1}^{-1}\left(\operatorname{st}\left(p_{i-1}(x)\right)\right)$.

Since $r^{-1}(x)$ is not empty for every $x \in X, r$ is onto. Fix $x \in X$ and let us show that $r^{-1}(x)$ satisfies the conclusions of the theorem. Since $T=$ $\alpha_{i}^{-1}\left(\operatorname{st}\left(p_{i}(x)\right)\right)$ is $(n-1)$-connected we see that $r^{-1}(x)$ is approximately 
$(n-1)$-connected as the inverse limit of $(n-1)$-connected finite simplicial complexes.

Let a group $G$ be such that $\operatorname{dim}_{G} X \leq n$. Note that $H_{n}(T)$ is $\mathcal{F}$ torsion and $G$ is $\mathcal{F}$-torsion free. Then by the universal coefficient theorem $H^{n}(T ; G)=\operatorname{Hom}\left(H_{n}(T), G\right)=0$. Thus $\widetilde{H}^{k}\left(r^{-1}(x) ; G\right)=0$ for $k \leq n$ and since $\operatorname{dim}_{G} Z \leq n$, we have $\widetilde{\breve{H}}^{k}\left(r^{-1}(x) ; G\right)=0$ for $k \geq n+1$. Hence $r$ is $G$-acyclic and this completes the proof.

\section{References}

[1] A. K. Bousfield and D. M. Kan, Homotopy Limits, Completions and Localizations, Lecture Notes in Math. 304, Springer, Berlin, 1972.

[2] M. Cencelj and A. N. Dranishnikov, Extension of maps to nilpotent spaces, II, Topology Appl. 124 (2002), 77-83.

[3] A. N. Dranishnikov, Rational homology manifolds and rational resolutions, Topology Appl. 94 (1999), 75-86.

[4] A. Dranishnikov, Cohomological dimension theory of compact metric spaces, Topology Atlas Invited Contributions, http://at.yorku.ca/topology/taic.htm.

[5] A. Koyama and K. Yokoi, Cohomological dimension and acyclic resolutions, Topology Appl. 120 (2002), 175-204.

[6] M. Levin, Acyclic resolutions for arbitrary groups, Israel J. Math. 135 (2003), 193204.

[7] —, Cell-like resolutions preserving cohomological dimensions, preprint.

[8] - Universal acyclic resolutions for finitely generated coefficient groups, Topology Appl., to appear.

Department of Mathematics

Ben Gurion University of the Negev

P.O. Box 653

Be'er Sheva 84105, Israel

E-mail: mlevine@math.bgu.ac.il 